\title{
External Version of Laplace Method
}

\author{
Tahir H. Ismail \\ Department of Mathematics \\ College of Computer Sciences and Mathematics \\ University of Mosul, Mosul, Iraq
}

Copyright (C2014 Tahir H. Ismail. This is an open access article distributed under the Creative Commons Attribution License, which permits unrestricted use, distribution, and reproduction in any medium, provided the original work is properly cited.

\begin{abstract}
The aim of this paper is to use some concepts of nonstandard analysis introduced by Robinson A. and axiomatized by Nelson E. to give an external method of Laplace. More precisely under certain conditions we get the following result.

$$
\int_{0}^{\infty} e^{-\omega \phi(t)} \psi(t) d t=(1+\alpha) \frac{b \Gamma\left(\frac{s+1}{r}\right)}{r a^{\frac{s+1}{r}}} \frac{1}{\omega^{\frac{s+1}{r}}}
$$

Where $a, b, r, s$ are standard constants and $\alpha \simeq 0, \omega=\frac{1}{\alpha}$.

Keywords: Nonstandard analysis, Infinitesimal number, Unlimited real numbers.

\section{Introduction}

Throughout this paper the following definitions and notations will be used.

A real number $x$ is called unlimited if $|x|>r$ for all real $r>0$, [7].

A real number $x$ is called infinitesimal if $\frac{1}{x}$ is unlimited, [7].

Two real number $x$ and $y$ are said to be infinitely near, denoted by $x \simeq y$, if $x-y$ is infinitesimal [7].

A real number $x$ is called appreciable if it is neither unlimited nor infinitesimal, and the set of all positive appreciable real number is denoted by $A^{+}[2]$.

The collections of limited, unlimited real numbers and infinitesimals are said to be external sets ([1], [2], [6]).
\end{abstract}


The external set of infinitesimal real numbers only is called the monad of 0 , denoted by $m(0)$. In general the set of real numbers which are infinitely near to a standard real number $a$ is called the monad of $a$, denoted by $m(a),[3],[5]$.

The external set of limited real numbers is called the galaxy of 0 , denoted by gal(0). In general, if $a$ is a standard real number, then the set of real numbers $x$ such that $x-a$ is limited is called the galaxy of $a$, denoted by gal( $a)$ [4], [9].

If $\varepsilon>0$, the external set of real numbers $\alpha$ such that $\frac{\alpha}{\varepsilon} \simeq 0$ is called the $-m(\alpha)$, and it is strictly included $m(0)$ [8], [9].

If $\varepsilon \simeq 0$, the external set of real numbers $\mu$ such that $\frac{\mu}{\epsilon^{n}} \simeq 0$ for all standard $n \in \mathbb{N}$ is called $\varepsilon$ - micromonad, denoted by $\varepsilon-M$, [2], [6].

If $x$ is a limited point of $\mathbb{R}^{n}$ ( $n$ standard), then it is infinitly near to a unique standard point of $\mathbb{R}^{n}$, this unique point is called the standard part of $x$, or the shadow of $x$, denoted by $\operatorname{st}(x)$ or $0_{x}$ [2].

If $A$ is a subset of $\mathbb{R}^{n}$ ( $n$ standard), then shadow of $A$, denoted by ${ }_{A}^{\circ}$, is defined as the unique standard subset of $\mathbb{R}^{n}$ obtained by taking the standardization of the collection of shadows of the limited points of $A$, ([4], [6]).

Any set or formula in internal set theory, denoted by IST, is called internal in case it does not involve the predicate "standard", otherwise it is called external $([4],[5])$.

A function $f: A \rightarrow B$ is called an internal function if $A$ is an internal set ([2]).

A function $f:[a, b] \rightarrow[a, b]$ is called piecewise continuous on $[a, b]$ if

(i) When ever $x \simeq a^{+}$and $x \simeq t^{+}$implies $f(x)$ exists

(ii) $f$ is continuous at all, but a finite number, of points in $(a, b)$.

(iii) Left and right limits exist at all points of $(a, b)$. [9]

A standard function $f: \mathbb{R} \rightarrow \mathbb{R}$ is called s-continuous at the standard point $x$ in $\mathbb{R}$, if for all $y(x \simeq y \rightarrow f(x) \simeq f(y)),([2],[8])$.

If $\alpha \simeq 0$ and $x$ is a real number, we define the $\alpha-m(x)$ as follows:

$\alpha-m(x)=\left\{y \in \mathbb{R}: \frac{y-x}{\alpha} \simeq 0\right\}$

If $\alpha \simeq 0$ and $x$ is a real number, we define the $\alpha-\operatorname{gal}(x)$ as follows:

$\alpha-\operatorname{gal}(x)=\left\{y \in \mathbb{R}: \frac{y-x}{\alpha}\right.$ is limited $\}$

\section{The Main Result}

Theorem (2.1):

Let $\phi$ be an increasing standard function defined on $[0, \infty)$ such that $\phi(t)=$ $a t^{r}+\alpha(t) t^{r},(a, r>o, \alpha(t) \simeq 0$, for $t \simeq 0)$ and that $\phi(t) \geq m t^{q}(m, q>0)$ for certain $t \geq 0$.

Let $\psi$ be an internal piecewise continuous function defined on $(0, \infty)$ such that $\psi(t)=b t^{s}+\beta(t) t^{s},(b \neq 0, s>-1$ are standard, $\beta(t) \simeq 0$ for $t \simeq 0)$ and for 
each $d>0$ there exist standard constants $K, C$ such that

$$
|\psi(t)| \leq K e^{c \phi(t)} \text { for every } t \geq d
$$

Let $\omega$ be unlimited positive number, then

$$
\int_{0}^{\infty} e^{-\omega \phi(t)} \psi(t) d t=(1+\alpha) \frac{b \Gamma\left(\frac{s+1}{r}\right)}{r a^{\frac{s+1}{r}}} \cdot \frac{1}{\omega^{\frac{s+1}{r}}}, \alpha \simeq 0
$$

\section{Proof:}

Let $\delta \simeq 0$ such that

$$
\int_{0}^{\infty} e^{-\omega \phi(t)} \psi(t) d t \epsilon \frac{1}{\omega}-M
$$

Suppose that $\delta>\frac{1}{e^{\frac{1}{n}}}$ for every standard positive integer $n$.

Then

$$
\int_{0}^{\infty} e^{-\omega \phi(t)} \psi(t) d t=\int_{0}^{\delta} e^{-(a+\alpha(t)) t^{s}+\mu, \quad \mu=\frac{1}{\omega}-M}
$$

We observe that the passage from appreciable within infinitesimals for integrand workout when leaving the $\frac{1}{\omega^{\frac{1}{r}}}$-galaxy. We obtain an integrand, which is an $A^{+}$, near an integrand, whose integral is continuous. Putting $a t^{r}=u$, we get

$$
\int_{0}^{\infty} e^{-\omega \phi(t)} \psi(t) d t=\frac{b}{r a^{\frac{s+1}{r}}} \cdot \frac{1}{\omega^{\frac{s+1}{r}}} \int_{0}^{a \omega \delta} e^{-\left(a+\alpha\left(\frac{u}{a \omega}\right)^{\frac{1}{r}}\right) u^{\frac{s+1}{r}} d u+\mu}
$$

Put $I(a)=\left\{\begin{array}{cl}e^{-\left(a+\alpha\left(\frac{u}{a \omega}\right)^{\frac{1}{r}}\right) u}\left(1+\beta\left(\frac{u}{a \omega}\right)\right)^{\frac{1}{r}} u^{\frac{s+1}{r}} & \text { if } u \leq a \omega \delta^{\prime} \\ 0 & \text { if } u>a \omega \delta^{\prime}\end{array}\right.$

Then for every $u \in A^{+}$, we have

$$
I(u) \simeq u^{\frac{s+1}{r}}
$$

Also tor every $u \in \mathbb{R}^{+}$we have

$$
I(u) \leq 2 e^{\frac{-u}{2}} u^{\frac{s+1}{r}}
$$

By using proposition (2) ([8] , [13]), we obtain

Consequently

$$
\int_{0}^{\infty} I(u) d u \simeq \int_{0}^{\infty} e^{-u} u^{\frac{s+1}{r}} d u=\Gamma\left(\frac{s+1}{r}\right)
$$

$$
\int_{0}^{\infty} e^{-\omega \phi(t)} \psi(t) d t=(1+r) \frac{b \Gamma\left(\frac{s+1}{r}\right)}{r a^{\frac{s+1}{r}}} \cdot \frac{1}{\omega^{\frac{s+1}{r}}}+\mu \quad r \simeq 0
$$




$$
=(1+\alpha) \frac{b \Gamma\left(\frac{s+1}{r}\right)}{r a^{\frac{s+1}{r}}} \cdot \frac{1}{\omega^{\frac{s+1}{r}}}+\mu \quad \alpha \simeq 0
$$

\section{Remark (2.2):}

The assertion of the above theorem remains true if the functions $\varnothing$ and $\psi$ satisfy the conditions mentioned on a standard interval $[0, c), c>0$, and if we integrate from 0 to $c$.

We will assume that the function $\phi$ and $\psi$ satisfy always the always the conditions of theorem 2.1. Consider the following examples as illustration of the theorem 2.1 .

\section{Example ( 2.3):}

(1) If $\phi(t)=t$, and if $\psi(t)=b t^{n}+\beta(t) t^{n}$, with $n \in I N$ is standard we get

$$
\int_{0}^{\infty} e^{-\omega \phi(t)} \psi(t) d t=(1+\alpha) \frac{b n !}{\omega^{n+1}}, \alpha \simeq 0
$$

(2) suppose that $\phi$ is twice derivable at 0 , and that $\phi(t)=\frac{1}{2} \phi^{\prime \prime}(0) t^{3}+\alpha(t) t^{2}, \phi^{\prime \prime}(0)>0$. If $\psi(0) \simeq 0$ and that $\psi$ is $\mathrm{s}-$ continuous at 0 , then, noting that $\phi$ passed $a$ minimum vale at 0 .

$$
\int_{0}^{\infty} e^{-\omega \phi(t)} \psi(t) d t=(1+\alpha) \psi(0) \sqrt{\frac{2 \pi}{\phi^{\prime \prime}(0)}} \frac{1}{\sqrt{\omega}},(\alpha \simeq 0)
$$

The relation remains valid if we integrate over a standard interval $[c, d]$ such that $c<0$ and $d>0$.

(3) From the result of example (2) we deduce the stirling formula.

Because if $\omega \in \mathbb{N}$ is unlimited, we get

$$
\omega !=\int_{0}^{\infty} e^{-s} s^{\omega} d s=\int_{0}^{\infty} e^{-s+\omega \log s} d s
$$

We will bring back the last integral to are of the given in example (2). To identify the maximum value of the integral of $e^{-s+\omega \log s}$ at $\omega$, put $s=\omega+$ $\omega t$, then

$$
\begin{gathered}
\omega !=\int_{-1}^{\infty} e^{-s \omega-\omega \log \omega+\log (1+\omega)} d t \\
\omega !=\omega^{\omega} e^{\omega} \omega \int_{-1}^{\infty} e^{-\omega(t-\log (1+t))} d t \\
=\omega^{\omega} e^{\omega} \sqrt{\omega} \sqrt{2 \pi}(1+\xi) \quad \xi \simeq 0
\end{gathered}
$$

(4) We return to integrals of type given in example (1) but with nonstandard $n \in \mathbb{N}$, and determine the principle part of the integrals of type 


$$
\int_{0}^{\infty} e^{-\omega t} t^{v} g(t) d t
$$

where $\frac{v}{\omega}$ is limited and the function $g$ is internal, $s$-contiunous and appreciable on $\mathrm{gal}^{+}(0)$, and such that there exist standard constants $K, C$ such that $g(t) \leq K e^{c t}$ for every $t \geq 0$.

We notice that the maximum factor to fast variation $e^{-\omega t} t^{v}$ is reached for $=\frac{v}{\omega}$, we return to an integral of the type given in example (2) by putting $t=\frac{v}{\omega}+$ $\frac{v}{\omega} u$. Then

$$
\begin{gathered}
\int_{0}^{\infty} e^{-\omega t} t^{v} g(t) d t=\int_{-1}^{\infty} e^{-v(-v u+v(\log v-\log \omega+\log (1+u))} g\left(\frac{v}{\omega}+\frac{v}{\omega} u\right) \frac{v}{u} d u \\
=\frac{v e^{-v} v}{\omega^{v+1}} \int_{-1}^{\infty} e^{-v(u-\log (1+u))} g\left(\frac{v}{\omega}+\frac{v}{\omega} u\right) \frac{v}{u} d u
\end{gathered}
$$

Since $\frac{v}{\omega}$ is limited $g\left(\frac{v}{\omega}\right) \simeq 0$ and $g\left(\frac{v}{\omega}+\frac{u v}{\omega}\right)$ is s-continuous function of $u$ for every limited $u \geq 1$, then from the formula of example (2) and the striting formula we deduce that

$$
\begin{gathered}
\frac{v^{v} e^{-v} v}{\omega^{v+1}} \int_{-1}^{\infty} e^{-v(u-\log (1+u))} g\left(\frac{v}{\omega}+\frac{v}{\omega} u\right) d u \\
=(1+\propto) \frac{v^{v} e^{-v} \sqrt{v} \sqrt{2 \pi}}{\omega^{v+1}} g\left(\frac{v}{\omega}\right) \\
=(1+\xi) \frac{v !}{\omega^{v+1}} g\left(\frac{v}{\omega}\right)
\end{gathered}
$$

\section{Remark (2.3):}

We notice that as in the case of example (1) where the factor $e^{-w t} t^{v}$ has its maximum value contributes to a sensible manner the value of the integral $\int_{0}^{\infty} e^{-\omega t} t^{v} g(t) d t$

(5) Let $\phi$ be uninternal function such that (i) $\phi$ is $s-$ continuous on the $\mathrm{gal}^{+}(0)$. (ii) $\phi \simeq 0$ on $\mathrm{gal}^{+}(0)$. (iii) there exists standard constants $K$ and $C$ such that $\phi(t) \leq K e^{c t}$ for every $t \geq 0$. Let $\varepsilon \simeq 0$ and $\omega \in I R^{+}$be unlimited such that $\varepsilon w$ is limited. Then

$$
\int_{0}^{\infty} e^{-t} t^{\omega} \phi(\varepsilon t) d t=\Gamma(\omega+1) \phi(\varepsilon \omega)(1+\xi) \xi \simeq 0
$$

\section{Remark (2.4):}

The proof of the above formula consists of (a) where $\omega$ is unlimited, and return to the previous example by putting $s=\varepsilon t$, (b) in the case where $\omega$ is limit- 
ed the above formula is deduced from example (1). Observing that $\phi(0) \simeq(\varepsilon \omega)$.

\section{References}

[1] M. Davis, Applied Nonstandard Analysis, New York, John Wiley and sons, 1977.

[2] F. Diener and M. Diener, Nonstandard Analysis in Practice, Springer-Verlag, Berlin, Heidelberg, 1995.

[3] H.J. Keisler, Elementary Calculus: An infinitesimal Approach 2ed, California USA, 2005, pp. 30,36,28.

[4] T.H. Ismail, H. Y. Saleh, and B.M. Sulaiman, Characterizing Internal and External Sets, Iraqi Joumal of Statistical Science, 11(20) (2011), 63-69.

[5] T.H. Ismail, On the Extremes of Collection of External Numbers, International Journal of Enhanced Research in Science Technology \& Engineering, 3 (1) (2014), 37-41.

[6] LUJZ and M. Goze, Nonstandard Analysis , Practical Guide with Applications, Lecture Notes in Mathematics No. 881, Springer-verlag, 1981.

[7] E. Nelson, Internal set theory, A new approach to non standard analysis, Bull. of Amer. Math. Soc., 83 (6) (1977), 1165-1108.

[8] A. Robinson, Nonstandard Analysis, Revised Edition, Princeton University press, 1996.

[9] K.D. Stroyan and W.A. Luxemburg, Introduction to The Theory of Infinitesimal, New York, Academic Press, 1976.

Received: May 15, 2014 